\title{
The quadriceps active ratio: A dynamic MRI based assessment of patellar height
}

\author{
Peter McEwen ${ }^{1}$, Sergio Barroso Rosa ${ }^{1}$, Kaushik Hazratwala1, Matthew Wilkinson ${ }^{1}$, Brent Matthews ${ }^{1}$, Zaid Bahho ${ }^{1}$ \\ 1 The Orthopaedic Research Institute of Queensland, Townsville, Australia
}

Objective: Precise and accurate surgical correction of Patella Alta (PA) requires both a diagnosis of the condition and a target for correction. The radiographic ratios widely used for assessing patella height are flawed for a number of reasons. The Patellotrochlear Index (PTI) addresses some of these deficiencies but is done under passive conditions and in Bierdet's paper was not related to the diagnosis of PFI. We describe a quadriceps active modification of the $P T I$, the quads active ratio $(Q+R)$ and assess its reliability and accuracy as a test for the diagnosis of PFI due to PA.

Methods: One hundred patients undergoing a clinically indicated knee MRI scan were recruited into the study. Participants had an additional sagittal T1 sequence acquired with maximal static quadriceps contraction. Six scans were technically inadequate. The remaining 94 scans were screened for trochlear dysplasia and 16 scans were subsequently discarded so PFI could be assessed solely in terms of PA. There were 9 cases of PFI in the remaining 78 scans. In 56 of these scans a lateral radiograph was also available. The PTI and Q+R were calculated from MRI scans and the Insall Salvati (IS), Modified Insall-Salvati (MIS), Blackburn Peel (BP) and Caton-Deschamp (CD) ratios from lateral radiographs by 3 surgeons at 3 times points. The inter- and intra-rater reliability of the measures were calculated using intra-class correlation coefficient. Receiver operator curves were constructed to determine the ideal cut-off for each MRI based test for the diagnosis of PFI. Sensitivity and specificity of the MRI based tests for PFI was compared to the IS, MIS, BP and CD ratios using their previously published cut-offs.

Results: A main effect of time was found for the $P T I(P<0.01)$ but not for the $Q+R(P=0.052)$. A time $x$ surgeon interaction effect was shown for both the $P T I$ and the $Q+R(P<0.01$, respectively). However, one-way ANOVA at time point 3 showed no main effect of surgeons for either the PTI $(p=0.05)$ or $Q+R(p=0.09)$. The Intra-class Correlation Coefficient between surgeons for the $P T I$ and the $Q+R$ ranged from $0.65-0.75$ and $0.69-0.83$ respectively. The coefficient of variation between surgeons for the $\mathrm{PTI}$ and the $\mathrm{Q}+\mathrm{R}$ ranged from $0.88-9.88 \%$ and 2.24-4.85\% respectively. According to the area under the receiver operator characteristics curve, the $Q+R$ (AUC, 0.76; SE, 0.06; $95 \% \mathrm{Cl}, 0.87-0.64 ; \mathrm{P}<0.01$ ) was more accurate to detect PFI than the PTI (AUC, 0.74; SE, 0.06; $95 \% \mathrm{Cl}, 0.86-0.62 ; \mathrm{P}<0.05)$. The $\mathrm{Q}+\mathrm{R}$ and $\mathrm{PTI}$ cut-off scores of 0.12 and 0.36 gave sensitivities of $79.2 \%$ and specificities of $55.0 \%$ and $50.0 \%$ respectively. Of the widely used radiographic indices the Insall-Salvati ratio was the most accurate.

Conclusion. The $\mathrm{Q}+\mathrm{R}$ is more accurate than the $\mathrm{PTI}$ for the diagnosis of patella alta causing $\mathrm{PFI}$ and addresses many of the deficiencies in other assessment methods. In the absence of trochlear dysplasia a $Q+R$ cut-off of 0.12 may be a reasonable target for correction of Patella Alta.

The Orthopaedic Journal of Sports Medicine, 5(5)(suppl 5)

DOI: $10.1177 / 2325967117$ S00193

(C)The Author(s) 2017

This open-access article is published and distributed under the Creative Commons Attribution - NonCommercial - No Derivatives License (http://creativecommons.org/licenses/by-nc-nd/3.0/), which permits the noncommercial use, distribution, and reproduction of the article in any medium, provided the original author and source are credited. You may not alter, transform, or build upon this article without the permission of the Author(s). For reprints and permission queries, please visit SAGE's Web site at http://www.sagepub.com/journalsPermissions.nav. 